\title{
Changes in visual function and in the posterior segment of the eye during treatment of onchocerciasis with diethylcarbamazine citrate
}

\author{
A. C. BIRD, ${ }^{1}$ HADI EL SHEIKH, ${ }^{2}$ J. ANDERSON,* AND H. FUGLSANG* \\ From the ${ }^{1}$ Department of Clinical Ophthalmology, Institute of Ophthalmology, \\ Moorfields Eye Hospital, City Road, London ECIV 2PD, and the \\ ${ }^{2}$ Khartoum Eye Hospital, Khartoum, Democratic Republic of Sudan
}

SUMMARY Eighteen patients, moderately to heavily infected with onchocerciasis, were treated with a standard course of diethylcarbamazine citrate (DEC-C). In addition to the well recognised systemic reactions to treatment, the following ocular changes occurred during therapy: (1) Transient pigment epithelial lesions were detected in 7 patients by fluorescein angiography. (2) Optic disc leakage, as shown by fluorescein angiography, developed in 9 patients, and increase of pre-existing leakage was demonstrated in 3 further patients. (3) Visual field loss was documented in 5 patients. These observations have grave implications for DEC-C therapy in onchocerciasis. They emphasise the need for great caution in its use in the treatment of onchocerciasis in heavily infected patients and for close supervision of ocular function during therapy.

It is generally agreed that the clinical manifestations of onchocerciasis are related to the presence throughout the body of microfilariae and that the adult worms (macrofilariae) play little part in the production of disease save as a source of microfilariae. Since its introduction over 30 years ago diethylcarbamazine citrate (DEC-C) has been the principal microfilaricide in the treatment of onchocerciasis. It may be used alone or given as an adjunct to suramin, which is used mainly as a macrofilaricide but is also microfilaricidal. It is generally agreed that DEC causes severe systemic reactions during treatment of heavily infected patients, such as dermatitis, lymphadenopathy, headache, and vertigo, and these have been the principal objections to it (Fuglsang and Anderson, 1974. Bryceson et al., 1977). It has been suggested that vision may be seriously impaired, and in particular there is some evidence that optic atrophy may be precipitated by the use of DEC (Anderson et al., 1976a; Anderson and Fuglsang, 1978).

The present study was designed to assess if visual loss and morphological changes in the posterior segment of the eye occur with DEC therapy.

\footnotetext{
*World Health Organisation consultants.

Correspondence to Professor Bird.
}

\section{Patients and methods}

The patients were drawn from villages on the Bussere river, near the town of Wau in Bahr el Ghazal Province of Southern Sudan, where moderate to heavy infection with Onchocerca volvulus had already been identified by previous survey (Zaroug, Bashir, Fuglsang and Anderson, in preparation). Patients who conformed to the following criteria were invited to join the study: (i) visual acuity of $6 / 6$ with each eye, or, if worse, that the loss was due to disease other than onchocerciasis; (ii) no gross choroidoretinal disease; (iii) visual field loss restricted to arcuate defects; (iv) microfilariae detected in the anterior ocular tissues; (v) clear ocular media.

Eighteen male patients were admitted to an 8-day therapeutic study. Only 4 had received previous treatment for onchocerciasis: patient 1 had injections of suramin in 1969, patient 3 in 1978, patient 7 in 1974, and patient 9 many years previously. Three other male patients were treated with a shorter, more intensive course of DEC-C.

Before the start of treatment a general examination was undertaken, including assessment of nodules. Skin snips were taken from the buttock and near the outer canthus of the eye with a corneoscleral punch, and microfilarial counts were made by standardised techniques (Fuglsang and Anderson, 1977). 
Visual acuity was measured at 6 metres by an illiterate ' $E$ ' chart, and visual fields were assessed by confrontation. New field defects were identified if loss was detected in a previously intact superior or inferior hemifield. Changes of a pre-existing field defect could not be judged with certainty by this technique and were not recorded as a change. Pupils were tested by the 'swinging flash-light test'.

The number of globular infiltrates (Jones et al., 1978) at the limbus was counted each day by means of a loupe and focal illumination. The anterior segments were examined with a Haag-Streit slitlamp, and an assessment was made of the number of microfilariae in the anterior chamber and cornea. Colour and fluorescein fundus photography was undertaken with a Kowa RC 2 fundus camera with the pupils dilated. Fluorescein angiograms were recorded on Ilford HP5 film (ASA 400) after intravenous injection of $20 \%$ fluorescein sodium.

\section{MANAGEMENT}

The patients were admitted to the Military Hospital, Wau, for treatment with DEC-C at a dosage schedule similar to that which has been employed routinely for the treatment of onchocerciasis. The first 18 patients were given $25 \mathrm{mg}$ b.d. on the first day, $50 \mathrm{mg}$ b.d. on the second, $100 \mathrm{mg}$ b.d. on the third, and $150 \mathrm{mg}$ b.d. on the 5 subsequent days, except patient 2, who was given half doses because of his small size $(28 \mathrm{~kg})$.

The patients' general status was assessed twice daily, and the level of treatment was reduced if there appeared to be excessive disability as a result of therapy. Skin snips were repeated on the seventh day of treatment. Visual function was recorded and fundus photography repeated on alternate days after the start of treatment. Slit-lamp examination was repeated on the fifth day of treatment.

\section{Results (see Table 1)}

\section{PRE-TREATMENT STATUS}

General

All 18 patients had palpable nodules in the pelvic region, and patient 12 had 1 on the head. Microfilarial counts at the buttock were over 100 per snip in all but 1 patient and at the outer canthus over 15 in 11.

\section{Ocular}

Microfilariae were seen in the cornea of all patients, and in the anterior chamber in all but 3 . All but 4 patients had a visual acuity of $6 / 6$ with each eye, and the worst vision recorded was 6/9. In 2 of them minor traumatic corneal scars were present, and in the other 2 no reason for the mild reduction in acuity was found. In patients 1-9 no visual field loss was detected; patients $10,11,13$, and 16-18 had arcuate loss in the superior and inferior hemifields of each eye; patients 12 and 14 had one intact hemifield; and patient 15 had field loss in 1 eye only.

Nine patients had normal fundi and angiograms (nos. 2, 4, 7, 8, 10, 12, 14-16), 6 had mild optic disc leakage on angiography (nos. 1, 3, 5, 9, 13, 17), 1 had mild retinal pigment epithelial changes giving rise to transmission defects (no. 6), and 2 had pigment epithelial defects and optic disc leakage (nos. 11, 18).

\section{RESPONSE TO TREATMENT \\ General}

All patients developed itching associated with a papular skin rash which evolved to confluent oedema by the third day of treatment, and which resolved in nearly all patients by the end of the fourth day. Similarly all had early tenderness of the inguinal and axillary lymph nodes, which started to resolve after 4 days of treatment. The severity of these symptoms caused us to reduce the dose during the second day of treatment in patients 2 and 14 . In patient 2 vomiting occurred immediately after taking the tablets on the morning of day 3 , attenuating further the level of treatment early in his course.

All but 6 patients (nos. 2, 3, 10, 11, 14, and 17) complained of headache, particularly during the first 4 days of treatment, and all but 4 had vertigo (nos. 6, 7, 11, and 14), which had a similar time course to the headache. A few patients had symptoms of upper respiratory tract inflammation and some had gastrointestinal disturbances.

During the second half of the course of treatment arthralgia was the most severe complication, affecting 10 patients (nos. 1, 3, 7, 8, 10-14, and 16), and it was particularly severe in nos. 3, 10,12, and 14. Most commonly involved were wrist, ankle, elbow, and metatarsophalangeal joints, and some patients complained of backache. In patient 10 the extent of the disability caused us to reduce the dose of DEC-C. Microfilarial skin counts on the seventh day at the buttock were 0 in 10 patients, 1 in 3 and $2-5$ in 5 , and at the outer canthus 0 in 13, 1 in 4 , and 2 in 1 .

\section{Ocular}

Globular infiltrates at the limbus occurred in all patients during the first day of treatment, and they were seen until the end of the course of DEC-C. There was considerable fluctuation in the number of globular infiltrates from day to day, and the number did not correlate with any other attribute of the patients' response. 
Table 1 Clinical manifestation of disease and new lesions acquired during DEC-C therapy

\begin{tabular}{|c|c|c|c|c|c|c|c|c|c|c|c|c|c|}
\hline \multicolumn{2}{|c|}{ Patients } & \multicolumn{4}{|c|}{ Pretreatment $M F$ count } & \multicolumn{2}{|l|}{$V A$} & \multicolumn{2}{|c|}{ Pre-existing lesions } & \multicolumn{4}{|c|}{ New lesions } \\
\hline No. & Age & $A C$ & $C$ & $O C$ & $\boldsymbol{B}$ & $R E$ & $L E$ & Field loss & $O D$ leak & Field loss & $R P E$ & $O D$ leak & Arthralgia \\
\hline 1 & 45 & 6-20 & $6-20$ & 144 & 471 & $6 / 6$ & $6 / 9$ & - & + & - & - & + & + \\
\hline 2 & 10 & $6-20$ & $20-50$ & 137 & 478 & $6 / 6$ & $6 / 6$ & - & - & - & + & + & - \\
\hline 3 & 25 & $50+$ & $20-50$ & 34 & 67 & $6 / 6$ & $6 / 6$ & - & + & $\stackrel{+}{(4,8 \text { days })}$ & + & ++ & ++ \\
\hline 4 & 33 & 1 & $50-100$ & 48 & 269 & $6 / 6$ & $6 / 9$ & - & - & - & + & + & - \\
\hline 5 & 40 & 1 & $50-100$ & 70 & 190 & $6 / 6$ & $6 / 6$ & - & + & $\stackrel{+}{\text { (8 days) }}$ & - & + & - \\
\hline 6 & 18 & 1 & $6-20$ & 4 & 228 & $6 / 6$ & $6 / 6$ & - & - & - & + & + & - \\
\hline 7 & 20 & 0 & $6-20$ & 2 & 172 & $6 / 6$ & $6 / 6$ & - & - & - & - & - & + \\
\hline 8 & 30 & 1 & $6-20$ & 3 & 257 & $6 / 6$ & $6 / 9$ & - & - & - & - & + & + \\
\hline 9 & 45 & 0 & $2-5$ & 2 & 553 & $6 / 6$ & $6 / 6$ & - & + & - & - & + & - \\
\hline 10 & 40 & 1 & $20-50$ & 43 & 627 & $6 / 6$ & $6 / 6$ & + & - & - & - & + & ++ \\
\hline 11 & 16 & 1 & $6-20$ & 190 & 119 & $6 / 6$ & $6 / 9$ & + & + & - & + & ++ & + \\
\hline 12 & 25 & $50+$ & $6-20$ & 120 & 223 & $6 / 6$ & $6 / 6$ & + & - & $\stackrel{+}{(2 \text { days })}$ & + & + & ++ \\
\hline 13 & 30 & 6-20 & $6-20$ & 1 & 600 & $6 / 6$ & $6 / 6$ & + & + & - & - & + & + \\
\hline 14 & 15 & $2-5$ & $50-100$ & 20 & 697 & $6 / 6$ & $6 / 6$ & + & - & $\stackrel{+}{\text { (4 days) }}$ & - & + & ++ \\
\hline 15 & 18 & $50+$ & $20-50$ & 18 & 360 & $6 / 6$ & $6 / 6$ & + & - & $\stackrel{+}{\text { (6 days) }}$ & - & + & - \\
\hline 16 & 28 & 0 & $2-5$ & 16 & 706 & $6 / 6$ & $6 / 6$ & + & - & - & - & + & + \\
\hline 17 & 26 & $50+$ & $2-5$ & 5 & 842 & $6 / 6$ & $6 / 6$ & + & + & - & + & ++ & - \\
\hline 18 & 39 & 1 & $2-5$ & 11 & $900+$ & $6 / 6$ & $6 / 6$ & + & + & - & - & + & - \\
\hline 19 & 31 & 1 & 0 & 8 & 223 & $6 / 18$ & $6 / 6$ & - & - & - & - & - & - \\
\hline 20 & 24 & 0 & $20-50$ & 83 & 500 & $6 / 6$ & $6 / 6$ & - & + & - & + & ++ & - \\
\hline 21 & 32 & 0 & $6-20$ & 98 & 689 & $6 / 6$ & $6 / 60$ & + & + & - & + & + & - \\
\hline
\end{tabular}

$\mathrm{AC}=$ anterior chamber. $\mathrm{C}=$ cornea. $\mathrm{OC}=$ outer canthus. $\mathrm{B}=$ buttock. $\mathrm{OD}=$ optic disc. $\mathrm{RPE}=$ retinal pigment epithelium. $+=\mathrm{Moderate}$. $++=$ Marked.

At 5 days nearly all the corneal microfilariae were straight, and there was opacification of the surrounding corneal stroma except in patients 7 and 10. In the anterior chamber live microfilariae were seen in patients $2,4,5,12,15,17$; there was a mild flare in most cases, but no cells were seen.

On day 2 patients $3,4,6,11,12$, and 17 showed discrete retinal pigment epithelial lesions at the posterior pole. They were undetectable by ophthalmoscopy and were demonstrated only by fluorescein angiography. In the dye transit there was no obvious abnormality, but during the subsequent 5 minutes multifocal progressive dye leakage developed into the retinal pigment epithelium, giving rise to multiple small hyperfluorescent spots at the posterior pole (Figs. 1-3). The distribution of these lesions was not constant, but there was a tendency for them to occur at the edge of the macula region (10-15 $5^{\circ}$ from the fovea). In all but patient 6 these lesions had resolved by day 4 . On day 4 patient 2 had these multifocal lesions (Fig. 1); it was this patient who had missed 2 doses of DEC-C, so that this did not represent the 4th day of constant therapy. After resolution there were no changes at the site of the previous retinal pigment epithelial lesions in some cases, while there were small transmission defects in others.

Optic disc leakage occurred in one or both eyes in 9 patients (nos. 2, 4, 6, 8, 10, 12, 14-16) in whom there was no dye leakage at the time of the initial study (Figs. 2 and 4), and in 3 (nos. 3, 11, 17) leakage increased when the initial and final studies were 

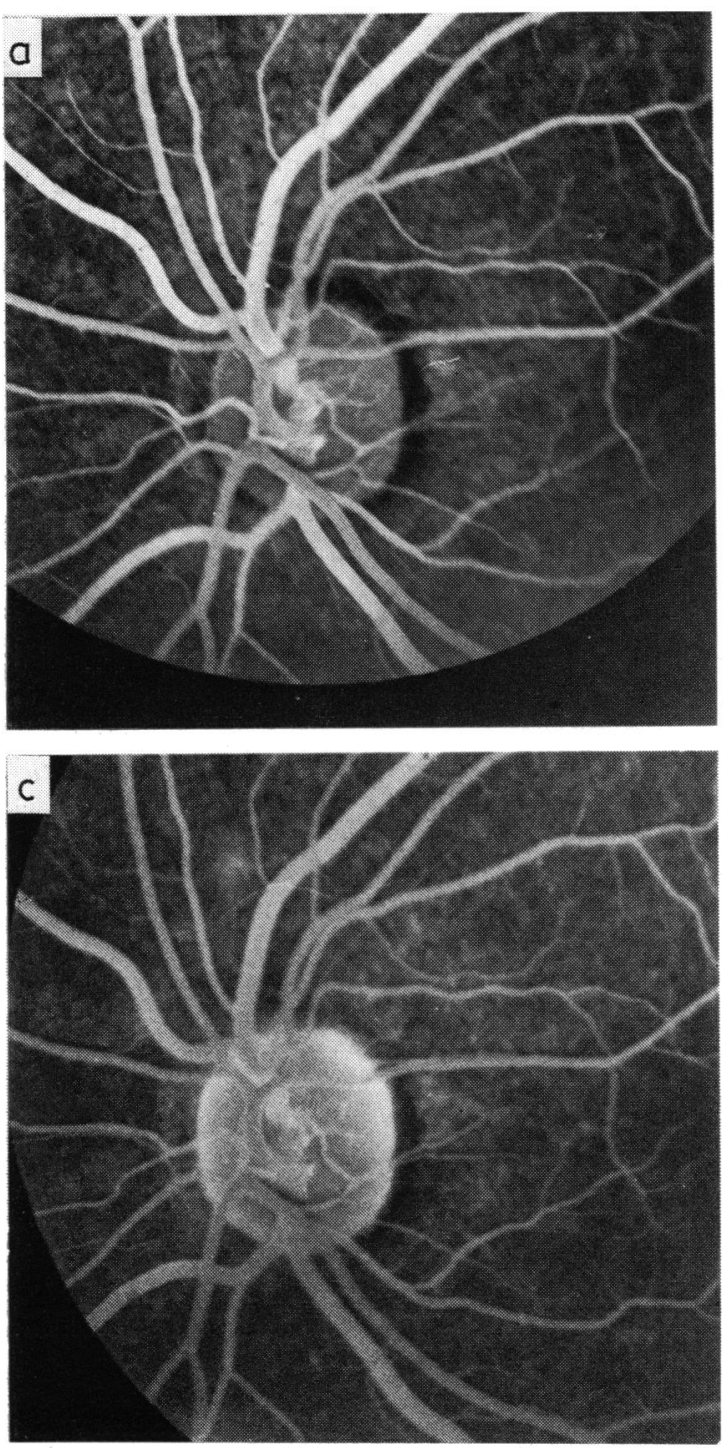

compared. In most patients the leakage progressed during the course of treatment but was confined to the optic disc. Dye leakage into the surrounding neuroretina occurred in only 2 patients (nos. 3,11 ) (Fig. 2).

No patient lost visual acuity. In 5 new visual field loss was recorded (nos. 3, 5, 12, 14, and 15) and in patient 3 this occurred in both eyes; this finding was recorded from 2 to 8 days after starting DEC-C. The defects were arcuate and progressed very rapidly, and in patients 3,12 , and 14 nearly a whole hemifield had been lost to hand movements by day 6 . At this stage it was considered undesirable to continue DEC-C, and patients 3,12, 14, and 15

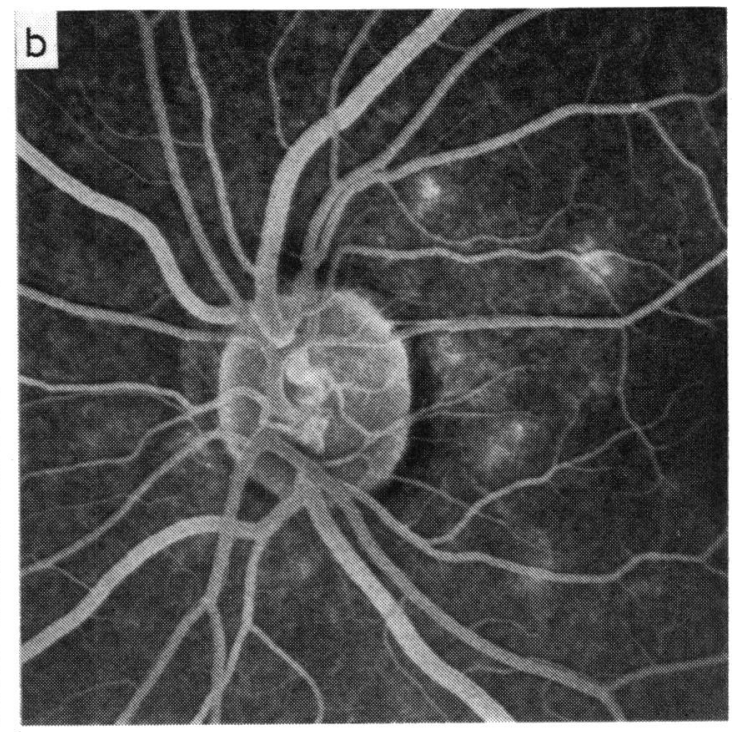

Fig. 1 Fluorescein angiogram on patient 2 which was normal on day 0 (a), showed mild pigment epithelial lesions on day 4 (b), and transmission defects and disc leakage on day $8(c)$

were given betamethasone $6 \mathrm{mg}$ daily for 2 days. The defects were not reversed, even though in patient 15 a defect had been present for no more than 2 days.

\section{SUBSIDIARY STUDY}

Three additional patients (nos. 19-21) were admitted for a short trial of DEC-C to observe in more detail the time course of the pigment epithelial lesions. In 2 (nos. 19, 20) there was no evidence of pre-existing ocular damage due to onchocerciasis, though patient 19 had a traumatic corneal scar in the right cornea with reduced acuity. Patient 21 had noticed poor left eye vision in early childhood. 

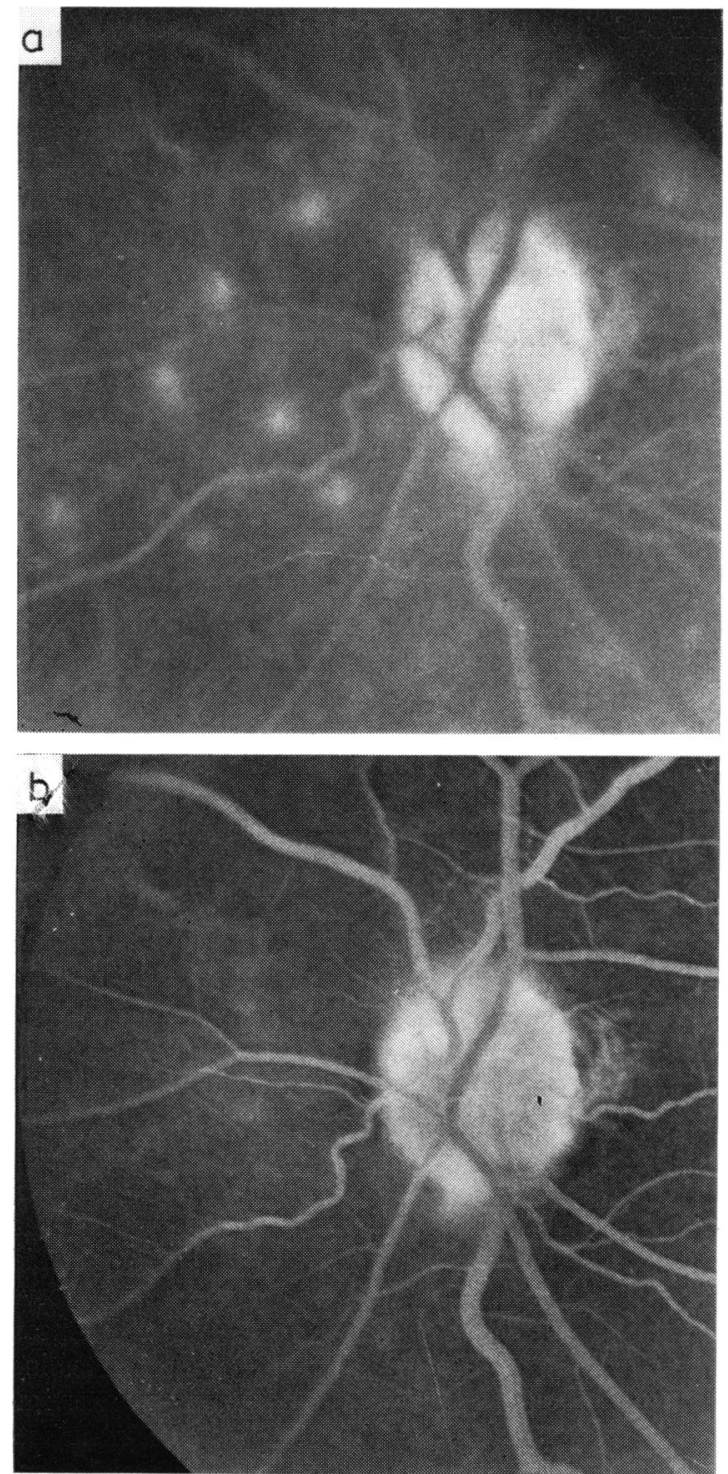

Fig. 2 Fluorescein angiogram on patient 3 showing pigment epithelial lesions around the optic disc leakage on day 2 (a). By day 8 the pigment epithelial lesions had resolved, but the disc leakage persisted and extended into the neuroretina (b)

This eye also had a corneal scar due to trauma, the eye was divergent, and there was evidence of left optic nerve disease in the form of visual field loss and a left relative afferent pupillary defect. Patient 19 had received injections of suramin and DEC tablets 4 years previously for onchocerciasis. All 3 were moderately to heavily infected and had ocular microfilariae.
These patients were given DEC-C $50 \mathrm{mg}$ b.d. on day $1,100 \mathrm{mg}$ b.d. on day 2 , and $150 \mathrm{mg}$ b.d. on day 3. All 3 suffered skin itching, headache, lymph node enlargement, and vertigo. The ocular status was reviewed daily during the 3 days of treatment. Patient 19 had no morphological changes in the fundus during the 3 days of review. Patient 20 developed pigment epithelial lesions on day 1 . These had resolved by day 2 , by which time new lesions had appeared (Fig. 5); they had all resolved
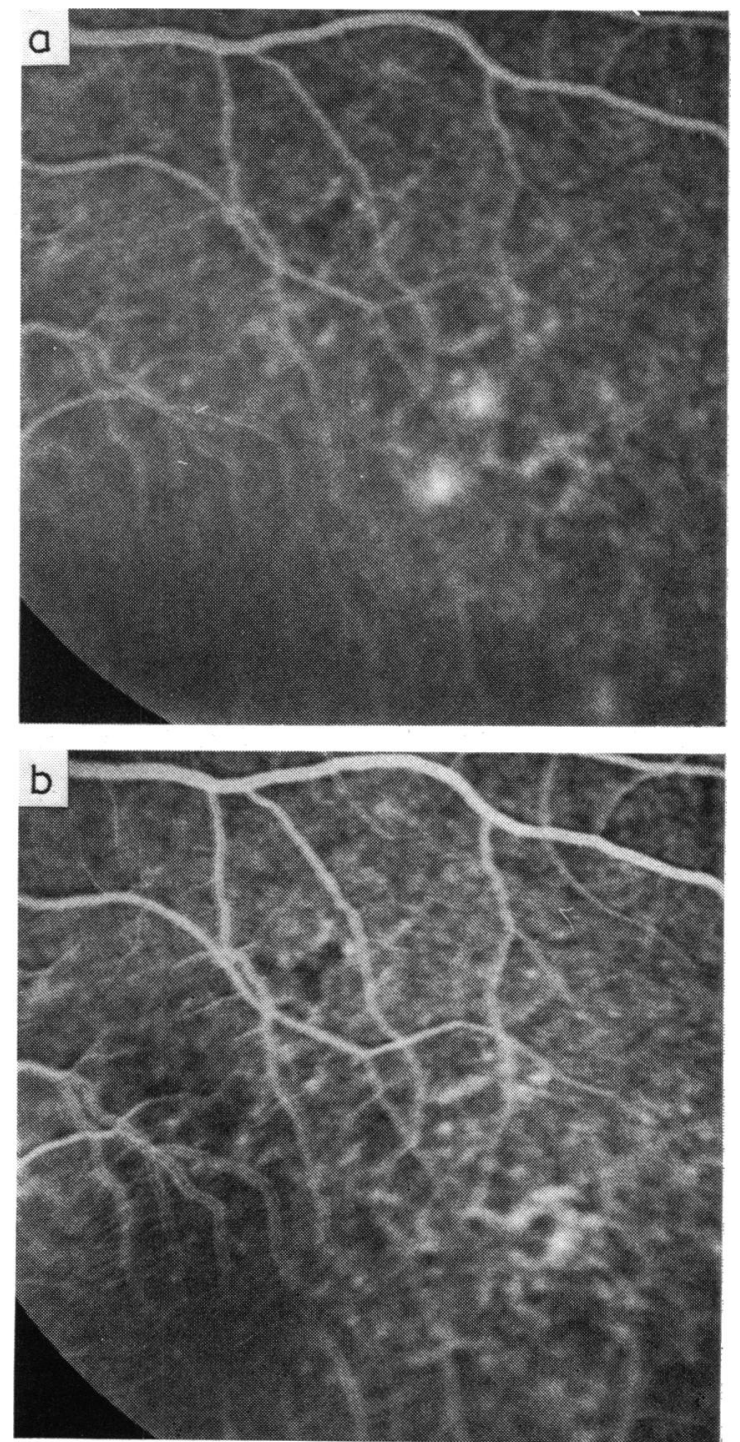

Fig. 3 Patient 11 had pigment epithelial lesions on fluorescein angiography and pre-existing transmission defects on day 2 (a). By day 6 the new pigment epithelial lesions had resolved $(b)$ 
by day 3 , but dye leakage at the optic disc was recorded at that time. No. 21 had pigment epithelial lesions on day 2 only, and the optic disc leakage increased.

\section{Discussion}

Since no control group was used in this study, it could be argued that the changes recorded in these patients occurred by chance and were unrelated to
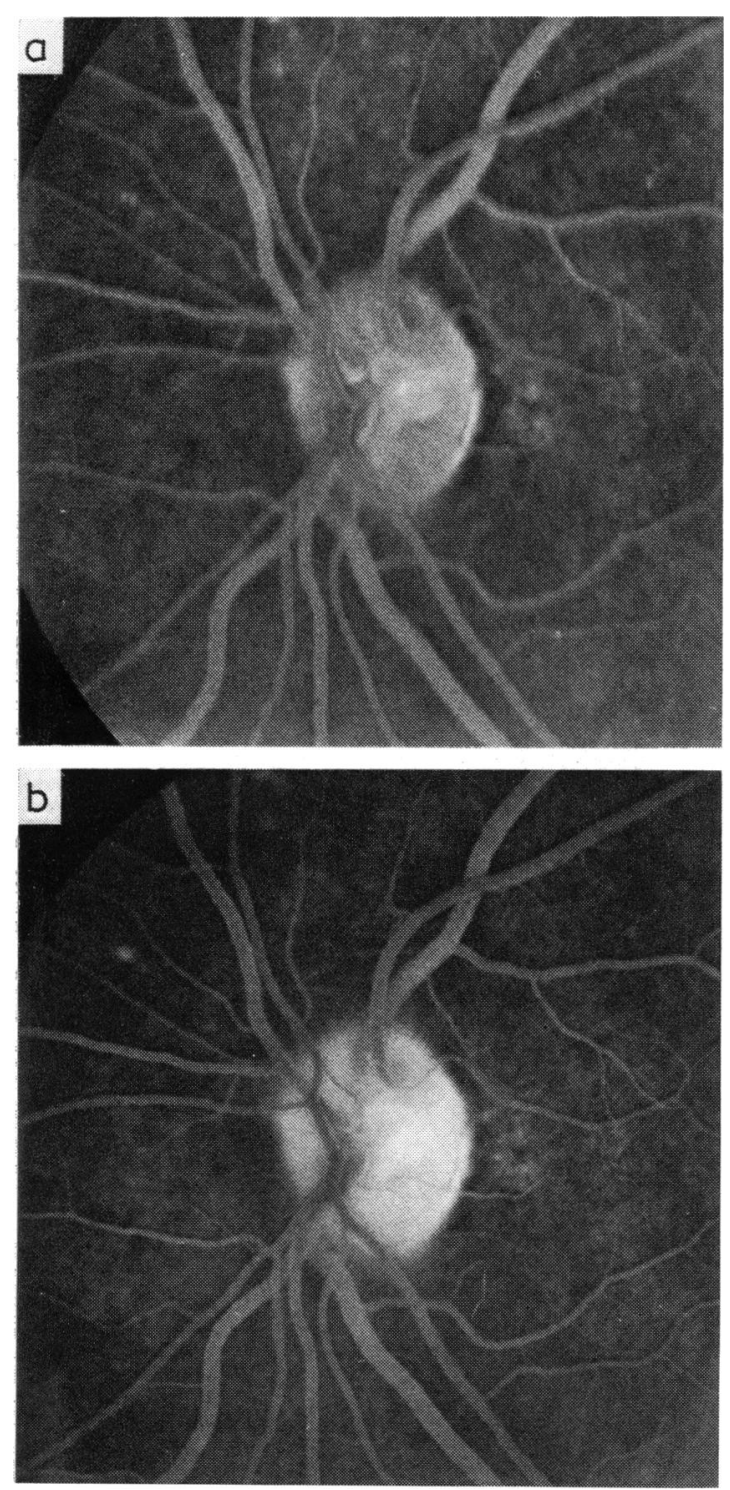

Fig. 4 Fluorescein angiography on patient 6 showing development of optic disc leakage from day 0 (a) to day 6 (b)
DEC-C therapy. However, the finding of change in so high a proportion of the patients argues against this view. Patients were selected for study on the basis of availability and were not exceptional when compared with other members of the community in which they lived (Zaroug et al., in preparation), and there is no reason to believe that they were particularly at risk of developing acute posterior segment inflammatory disease. Furthermore, no retinal pigment epithelial disease of the type recorded was seen before the start of therapy, and it was rarely observed in our previous studies (Bird et al., 1976). Similarly, the rapidity of visual field loss that occurred in our patients is unlikely to occur in the untreated disease. Therefore it is likely that the functional and morphological changes recorded were precipitated by DEC-C therapy.

The retinal pigment epithelial changes were short-lived in this group of patients and implied no obvious threat. They were presumably due to a transient injury early in the course of treatment. The time course of recovery is not exceptional for the retinal pigment epithelium, since it was identical to that seen after mild suprathreshold laser burns (Borland et al., 1978). The injury may have been caused by release of a toxic substance by the dying microfilariae. Alternatively choroidal inflammation may either have affected the pigment epithelium directly or have caused blood flow changes in the choriocapillaris, inducing temporary pigment epithelial ischaemia (Deutman and Lion, 1977; De Laey, 1978).

By contrast the optic nerve disease was sustained and appeared to be the cause of functional changes, since the pattern of visual loss clearly indicated anterior optic nerve disease. All patients with field loss had morphological changes at the optic nerve head, but several patients had disc changes without functional loss. Admittedly the method of testing for field loss was relatively crude, and it was impossible to detect with certainty changes in defects already present in the hemifield. It is possible that more sophisticated techniques would have revealed an even higher incidence of visual morbidy. The nature of the optic disc changes and the field loss resemble those in the untreated disease (Vedy and Sirol, 1971; Bird et al., 1976; Thylefors and Tøjum, 1978). Most authors agree that the optic nerve disease is inflammatory in origin, related to microfilariae which have been identified in the optic nerve (Mira, 1934; Rodger, 1960; Paul and Zimmerman, 1970), the inflammation being precipitated by microfilarial death.

There were no changes in retinal vascular permeability as shown by fluorescein angiography and no white spots beneath the internal limiting 

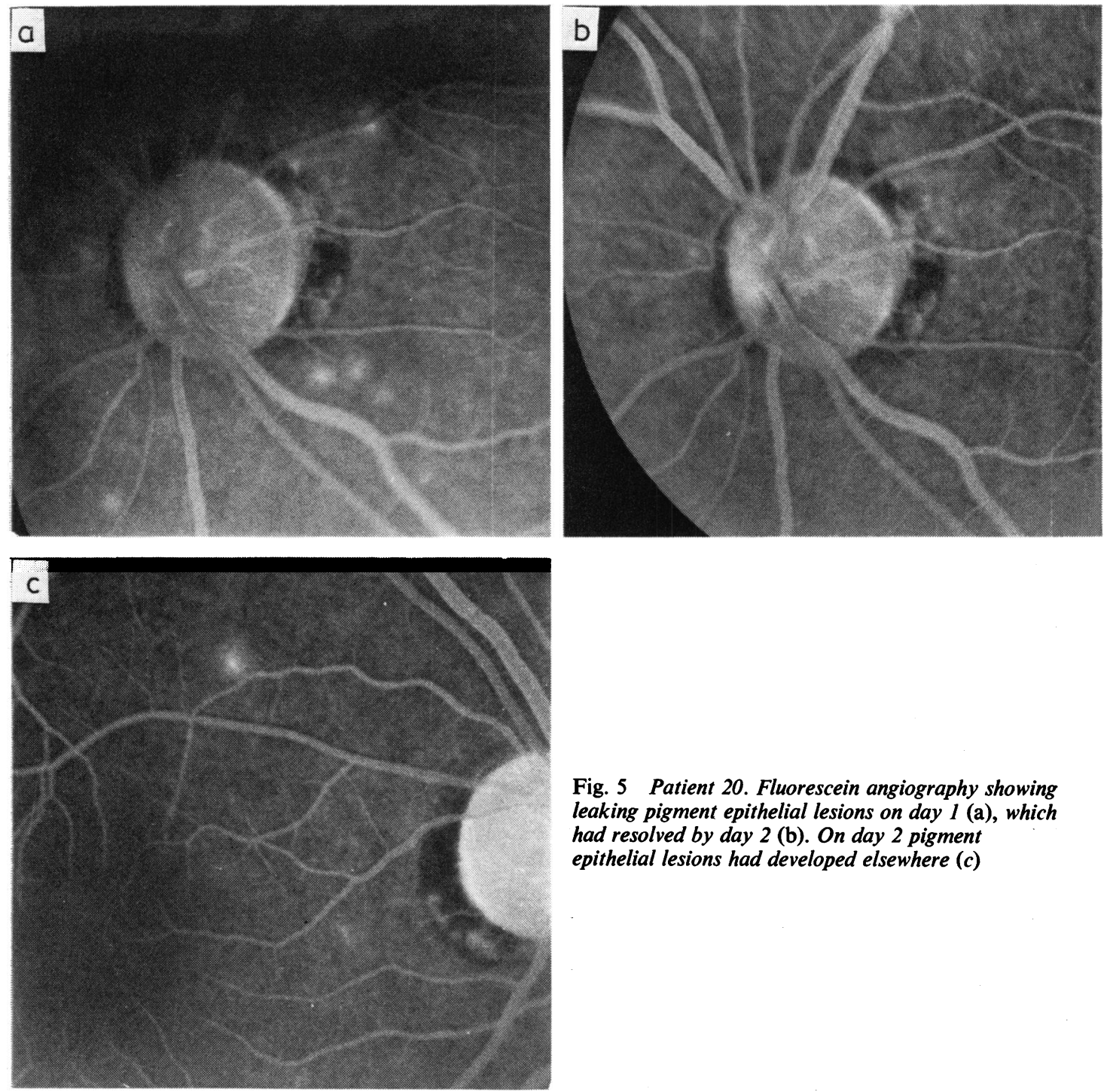

Fig. 5 Patient 20. Fluorescein angiography showing leaking pigment epithelial lesions on day 1 (a), which had resolved by day 2 (b). On day 2 pigment epithelial lesions had developed elsewhere (c)

membrane, as described by Vedy et al. (1971). The difference between our observations in Sudan and those in Chad (Vedy et al., 1971) may be related to the differences in the nature of the disease in different countries. Alternatively the lack of retinal changes may be due to a different effect of DEC-C on microfilariae in the choroid and optic nerve on the one hand and in the retina on the other. The presence of live microfilariae in the anterior chamber at a time when all corneal microfilariae were dead, as seen in our patient, would support this explanation.

The risk of posterior segment changes during DEC-C therapy appeared to be greater in patients with high corneal microfilarial counts, which would not be surprising if the changes detected were caused by dying microfilariae. The apparent correlation of DEC-C induced disease with the density of microfilariae in the ocular tissues would imply that studies on populations with different intensities of infection might provide different results. Patients more heavily infected than those described here may suffer a higher incidence of complications, whereas those more lightly affected may be at no risk from DEC-C therapy.

It was our impression that the most severe arthralgia occurred in those patients with visual field loss. Surprisingly, no other general pretreatment attribute distinguished those patients who suffered functional 
loss from the others and, apart from arthralgia, posterior segment inflammatory disease was not associated with any of the other manifestations of DEC-C therapy, such as severity of dermatitis, headache, vertigo, or the number of limbal globular infiltrates.

The number of ocular microfilariae detected did not appear to be the only determinant of posterior segment change leading to visual loss; patient 2 had a large number of microfilariae in the anterior chamber and cornea but had no functional loss, and there was nothing to indicate that he was at less risk than patient 14 . Other factors which may have influenced the effect of DEC-C, such as microfilarial mobilisation and immunological responses, were not studied in these patients.

Furthermore the results suggested that retinal pigment epithelial changes do not necessarily imply a threat of optic nerve disease and that optic nerve disease may occur in the patients without previously detected pigment epithelial changes. However, observations on patients 19-21 indicate that pigment epithelial changes may have resolved in some patients by day 2 at the time of the first review.

It is possible only to speculate on the pathogenetic relationship between DEC-C therapy and the changes recorded. It has been shown that DEC-C causes redistribution of microfilariae in the body tissues (Duke et al., 1976) and, more significantly, microfilarial destruction. Microfilarial death, whether spontaneous or as a result of DEC-C therapy, provokes inflammation in the cornea (Jones et al., 1978), and a marked increase of inflammation is seen in the epidermis during DEC-C administration (Rougemont et al., 1974). Inflammation appears to be an integral part of the therapeutic response to DEC-C therapy, and there is evidence to suggest that destruction of microfilariae is mediated by an immune response to surface antigens exposed by the action of DEC-C on the outer cuticle of the microfilaria (Rougemont et al., 1974; Gibson et al., 1976). It is unlikely that inflammation and optic nerve damage of the type reported here results from DEC-C alone, since they have not been described during animal toxicity studies (Harned et al., 1948) or during mass population therapy in the absence of onchocerciasis (Kessel et al., 1970; Che'en, 1964). It is generally agreed that the severe reactions during DEC-C treatment of onchocerciasis are related to destruction of microfilariae by the drug, and there is no evidence to suggest that microfilarial death induced by DEC-C is more likely to precipitate inflammation than spontaneous death, except in that the number of dying microfilariae after DEC-C is larger and therefore consequent response greater.
The pattern of disease seen in these patients receiving DEC-C resembled precisely the changes induced by onchocerciasis alone. The distribution of punctate retinal pigment epithelial lesions at the edge of the macula conformed with that seen before therapy and with that recorded in other parts of the world (Budden, 1973; Bird et al., 1976). Similarly, the optic disc swelling with limitation of hyperfluorescence to the disc tissues, and the arcuate field loss also mirrored the untreated disease state. The only differences were the rapidity of onset and time course of the processes. It was as if DEC-C had compressed several years' disease into a few days, which would support the concept that the disorder in either circumstance was related to microfilarial death.

The difference between the optic nerve and choroidal pigment epithelial disease in response to DEC-C therapy was striking. Following a survey of Bussere villages (Zaroug et al., in preparation) it was certainly our impression that the population from which our patients were drawn had a higher prevalence of optic nerve disease relative to choroidoretinitis (and sclerosing keratitis) than patients with onchocerciasis seen in other countries (Anderson and Fuglsang, 1977). It is possible that DEC-C therapy in communities elsewhere would provoke a different pattern of disease, and in particular that sustained choroidoretinitis might occur.

With such a short period of review the long-term effects of DEC-C treatment cannot be judged. It is possible that the functional deficit was temporary, but steroids, though given during a very short period, did not reverse the changes. Equally, it is possible that more patients may have suffered visual loss later than 8 days after starting DEC-C as a result of inflammation induced by treatment.

These observations, albeit on a small sample of patients from Southern Sudan without controls, have grave implications for DEC-C therapy in Sudan in particular and probably throughout the world and support the impressions gained as a result of previous studies (Anderson et al., 1976a, Anderson and Fuglsang, 1978). The course of DEC-C given in this study is in accord with current therapeutic practice and appears to carry a high visual morbidity due to posterior segment disease, but this should be set against the possible benefit to the patient derived from suppression of anterior segment disease following DEC-C therapy (Anderson et al., 1976a) and the visual loss which would occur in the untreated disease. Alternative therapeutic regimens, using long-term low-dose DEC-C, DEC-C combined with corticosteroids (Markell and Turner, 1957), or alternative microfilaricides, have been tried in the past in an attempt to avoid the 
intense discomfort of high-dose DEC-C (Rougemont et al., 1976). The apparent high risk to vision with DEC alone supports the rationale for seeking another way of controlling this disease, but any alternative therapeutic agent which causes massive microfilarial death may carry the same risk to vision in heavily infected patients unless it can be shown that changes detected in this study are somehow specific to DEC-C.

It is acknowledged that the action of suramin differs from that of DEC, and a similar study to that described here would be justified to assess its effect on the eye and the visual system. However, evidence exists to indicate that optic atrophy may follow treatment with suramin and that suramin therapy is also accompanied by severe systemic and ocular reactions (Anderson et al., 1976b). Such a response may be due to the microfilaricidal action of suramin (Duke, 1968), and Anderson and Fuglsang (1978) have suggested that an acceptable therapeutic regimen may require an initial course of DEC-C combined with corticosteroids followed by injection of suramin. If such a complex regimen proves safe and effective, there would be considerable logistic difficulties in delivering this treatment to large populations.

All these problems might be resolved if a therapeutic agent which was only macrofilaricidal existed which provided a means of eliminating adult worms from the body, allowing the microfilariae to die naturally during the ensuing 1-2 years. A single course of such a drug would be adequate in an area where there was Simulium control, but it would have to be repeated regularly where there was risk of reinfection. Clearly, patients heavily infected by $O$. volvulus present a major problem in management, and there is doubt whether the visual prognosis can be improved by any of the available therapeutic agents.

We thank Mr K. Sehmi for printing the photographs and Miss Julie Bramley for secretarial assistance. We are particularly grateful to Lieut. Col. Abuoaf for allowing us to use the facilities of the Military Hospital in Wau.

This study was supported by the Ministry of Health, Khartoum, with additional financial support from the Medical Research Council of Great Britain, and the filariasis component of UNDP/World Bank/WHO Special Programme for Research and Training in Tropical Diseases.

\section{References}

Anderson, J., and Fuglsang, H. (1977). Ocular onchocerciasis. Tropical Diseases Bulletin, 74, 257-272.

Anderson, J., and Fuglsang, H. (1978). Further studies on the treatment of ocular onchocerciasis with diethylcarbamazine and suramin. British Journal of Ophthalmology, 62, 450-457.

Anderson, J., Fuglsang, H., and Marshall, T. F. de C. (1976a). Effects of diethylcarbamazine on ocular oncho- cerciasis. Tropenmedizin and Parasitologie, 27, 263-278.

Anderson, J., Fuglsang, H., and Marshall, T. F. de C. (1976b). Effects of suramin on ocular onchocerciasis. Tropenmedizin und Parsitologie, 27, 279-296.

Bird, A. C., Anderson, J., and Fuglsang, H. (1976). Morphology of posterior segment lesions of the eye in patients with onchocerciasis. British Journal of Ophthalmology, 60, 2-20.

Borland, R. G., Brennan, B. H., Marshall, J., and Viveash, J. P. (1978). The role of fluorescein angiography in the detection of laser induced damage to the retina: a threshold study for Q-switched neodymium and ruby lasers. Experimental Eye Research, 27, 471-493.

Bryceson, A. D. M., Warrell, D. A., and Pope, H. M. (1977). Dangerous reactions to treatment of onchocerciasis with diethylcarbamazine. British Medical Journal, 1, 742-744.

Budden, F. (1973). Ocular onchocerciasis. American Journal of Ophthalmology, 76, 1027-1028.

Che'en, T. T. (1964). Demonstration of macrofilaricidal action of hetrazan, antimony and arsenic preparations in man. China Medical Journal, 83, 625-640.

De Laey, J. J. (1978). Fluoro-angiographic study of the choroid in man. Documenta Ophthalmologica, 45, 113-139.

Deutman, A. F., and Lion, F. (1977). Choriocapillaris nonperfusion in acute multifocal placoid pigment epitheliopathy. American Journal of Ophthalmology, 84, 652-657.

Duke, B. O. L. (1968). The effects of drugs on Onchocerca volvulus, 3 trials of suramin at different dosages and a comparison of the brands Antrypol, Moranyl and Naganol. Bulletin of the World Health Organisation, 39, 157-167.

Duke, B. O. L., Moore, P. J., and Vincelette, J. (1976). The population dynamics of Onchocerca volvulus microfilariae during treatment with suramin and diethylcarbamazine. Tropenmedizin und Parasitologie, 27, 133-144.

Fuglsang, H., and Anderson, J. (1974). Collapse during treatment of onchocerciasis with diethylcarbamazine. Transactions of the Royal Society of Tropical Medicine and Hygiene, 68, 72-73.

Fuglsang, H., and Anderson, J. (1977). The concentration of microfilariae in the skin near the eye as a simple measure of the severity of onchocerciasis in a community and as an indicator of danger to the eye. Tropenmedizin und Parasitologie, 28, 63-67.

Gibson, D. W., Connor, D. H., Brown, H. L., Fuglsang, H., Anderson, J., Duke, B. O. L., and Buck, A. A. (1976). Onchocercal dermatitis: ultrastructural studies of microfilariae and host tissues, before and after reatment with diethylcarbamazine (Hetrazan). American Journal of Tropical Medicine and Hygiene, 25, 74-87.

Harned, B. K., Cunningham, R. W., Halliday, S., Vessey, R. E., Yudia, N. N., Clark, M. C., Hine, C. H., Cosgrove, R., Subba Row, Y. (1948). Studies on the chemotherapy of filariasis. VI. Some pharmacodynamic properties of 1-diethylcarbamyl-4-methyl-piperazine hydroxide, hetrasan. Journal of Laboratory and Clinical Medicine, 33, 216235.

Jones, B. R., Anderson, J., and Fuglsang, H. (1978). Effects of different concentrations of diethylcarbamazine drops in ocular onchocerciasis. British Journal of Ophthalmology, 62, 428-439.

Kessel, J. F., Siliga, N., Tompkins, H., jr., and Jones, K. (1970). Recent studies on the control of filariasis in American Samoa. Journal of Parasitology, 56, 185-186.

Markell, E. K., and Turner, J. (1957). Cortisone and prednisolone in the suppression of allergic reactions to diethylcarbamazine treatment of onchocerciasis. American Journal of Tropical Medicine and Hygiene, 15, 546-552.

Mira, M. G. (1934). Sulla presenza delle microfilarie di 
'Onchocerca coecutiens Brumpt', nel nervo ottico. Riforma Medica, 50, 858, 861.

Paul, E. V., and Zimmerman, L. E. (1970). Some observations on ocular pathology of onchocerciasis. Human Pathology, 1, 581-563.

Rodger, F. C. (1960). The pathogenesis and pathology of ocular onchocerciasis. American Journal of Ophthalmology, 49, 560-590.

Rougemont, A., Boisson, M. E., and Zander, N. (1976). Première évaluation parasitologique d'une expérience de chimiotherapie collective après un an de traitement par la diethylcarbamazine dans un village d'hyperendémie onchocerquienne au Mali (Unpublished document, WHO/ ONCHO/76.125).
Rougemont, A., Discampts, G., and Boisson, M. E. (1974). Pathology of the cutaneous reaction and behaviour of $O$. volvulus microfilariae during treatment with diethylcarbamazine and suramin. Médecine Tropicale, 34, 508522.

Thylefors, B., and Tønjum, A. (1978). Visual field defects in onchocerciasis. British Journal of Ophthalmology, 62, 462-467.

Védy, J., and Sirol, J. (1971). A propos d'une papillite onchocerquienne. Médecine Tropicale. 31, 559-563.

Védy, J., Sirol, J., and Coulm, J. (1971). La rétinopathie ponctuée albescente onchocerquienne. Bulletin de la Société de Pathologie Exotique et de ses Filiales, 64, 471-486. 